\title{
X-ray facility for the characterization of the Athena mirror modules at the ALBA synchrotron
}

Dominique Heinis, Antonio Carballedo, Carles Colldelram, Guifré Cuní, Núria Valls Vidal, et al.

Dominique Heinis, Antonio Carballedo, Carles Colldelram, Guifré Cuní, Núria Valls Vidal, Óscar Matilla, Jordi Marcos, Alejandro Sánchez, Joan Casas, Josep Nicolàs, Nicolas Barrière, Maximilien J. Collon, Giuseppe Vacanti, Evelyn Handick, Peter Müller, Michael Krumrey, Ivo Ferreira, Marcos Bavdaz, "X-ray facility for the characterization of the Athena mirror modules at the ALBA synchrotron," Proc. SPIE 11852, International Conference on Space Optics - ICSO 2020, 1185222 (11 June 2021); doi: 10.1117/12.2599350

SPIE Event: International Conference on Space Optics - ICSO 2021, 2021, Online Only 


\section{International Conference on Space Optics-ICSO 2020}

Virtual Conference

30 March-2 April 2021

Edited by Bruno Cugny, Zoran Sodnik, and Nikos Karafolas
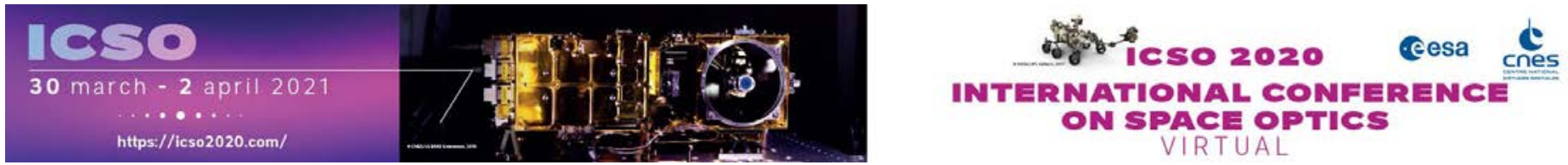

\section{$X$-ray facility for the characterization of the Athena mirror modules at the ALBA synchrotron}

\section{Cesa isoporecestings denes}




\title{
X-ray facility for the characterization of the ATHENA mirror modules at the ALBA synchrotron
}

\author{
Dominique Heinis ${ }^{\mathrm{a}}$, Antonio Carballedo ${ }^{\mathrm{a}}$, Carles Colldelram ${ }^{\mathrm{a}}$, Guifré Cunía ${ }^{2}$, Núria Valls \\ Vidal $^{\mathrm{a}}$, Óscar Matilla ${ }^{\mathrm{a}}$, Jordi Marcos ${ }^{\mathrm{a}}$, Alejandro Sánchez ${ }^{\mathrm{a}}$, Joan Casas ${ }^{\mathrm{a}}$, Josep Nicolàs ${ }^{\mathrm{a}}$, \\ Nicolas Barrière ${ }^{\mathrm{b}}$, Maximilien J. Collon ${ }^{\mathrm{b}}$, Giuseppe Vacanti ${ }^{\mathrm{b}}$, Evelyn Handick ${ }^{\mathrm{c}}$, Peter Müller ${ }^{\mathrm{c}}$, \\ Michael Krumrey ${ }^{\mathrm{c}}$, Ivo Ferreira ${ }^{\mathrm{d}}$, and Marcos Bavdaz ${ }^{\mathrm{d}}$ \\ ${ }^{a}$ ALBA Synchrotron, Carrer de la Llum 2-26, 08290 Cerdanyola del Vallès, Spain \\ ${ }^{\mathrm{b}}$ cosine measurement systems, Oosteinde 36, 2361 HE Warmond, The Netherlands \\ 'Physikalisch-Technische Bundesanstalt (PTB), Abbestr. 2-12, 10587 Berlin, Germany \\ dEuropean Space Agency, ESTEC, Keplerlaan 1, PO Box 299, 2200 AG Noordwijk, The \\ Netherlands
}

\begin{abstract}
The ALBA synchrotron ${ }^{1}$ (Barcelona, Spain) is building MINERVA a new X-ray beamline designed to support the development of the ATHENA mission (Advanced Telescope for High Energy Astrophysics). The beamline design is originally based on the monochromatic pencil beam XPBF $2.0^{2}$ at the Physikalisch-Technische Bundesanstalt (PTB), at BESSY II. MINERVA will provide metrology capabilities to integrate stacks produced by cosine company into a mirror module (MM) and characterize them. It will provide photons with a fixed energy of 1.0 $\mathrm{keV}$ with a residual divergence below $1 \times 1 \operatorname{arcsec}^{2} \mathrm{rms}$. The beam dimensions at the mirror module is adjustable from $10 \times 10 \mu \mathrm{m}^{2}$ up to $8 \times 8 \mathrm{~mm}^{2}$. Interoperability between MINERVA and XPBF 2.0 will be preserved in order to reinforce and boost the production and characterization of the mirror modules. MINERVA is funded by the European Space Agency (ESA) and the Spanish Ministry of Science and Innovation. Still in the detailed design phase, MINERVA will take 2 years to be completed for operation in 2022.
\end{abstract}

Keywords: X-ray optic, Silicon Pore Optics, Synchrotron, Metrology

\section{INTRODUCTION}

The ATHENA telescope is a space observatory that will address fundamental questions about energetic objects ${ }^{3}$ (accretion disk around black holes, large-scale structure, etc...). Located at a L2 slot, the telescope will be equipped with instrumentation able to perform both imaging and spectroscopy in the photon energy range from $0.2 \mathrm{keV}$ to $12 \mathrm{keV}$. One of the key elements of the telescope is the innovative modular architecture of its optics. In Figure 1 a) is shown the principal optical element of ATHENA made of 15 concentric rings and filed by about 700 sub-systems called mirror modules (MMs). Individual MM, in turn, is also organized in a set of four stacks of 37 highly polished silicon wafer reflecting plates. The technology used to manufacture the stacks is based on the Silicon Pore Optics technology developed at $\operatorname{cosine}^{4}$ (Figure 1 b)). Once completely manufactured, the whole set of assembled mirror modules will constitute a modified Wolter-Schwartzschield optics geometry with $12 \mathrm{~m}$ focal length.

The alignment of the 4 stacks constituting a singular MM is crucial to achieve the expected angular resolution of ATHENA. ${ }^{5}$ For that reason a dedicated X-ray metrology facility is required for their assembly and characterization. Today, at XPBF 2.0 (BESSY II in Berlin), cosine is continuously improving and seeking the adequate methodology to produce MMs at large scale. The current characterization method is briefly sketched in Figure $1 \mathrm{c})$ and d) and more details can be found in. ${ }^{6}$ In Figure $1 \mathrm{c}$ ) is presented a jig populated with 4 stacks. Three of them are rigidly attached to a dedicated hexapod while the last one remains motionless. The jig allows then

Further author information: (Send correspondence to Dominique Heinis.)

Dominique Heinis.: E-mail: dheinis@cells.es, Telephone: +34 935924052 


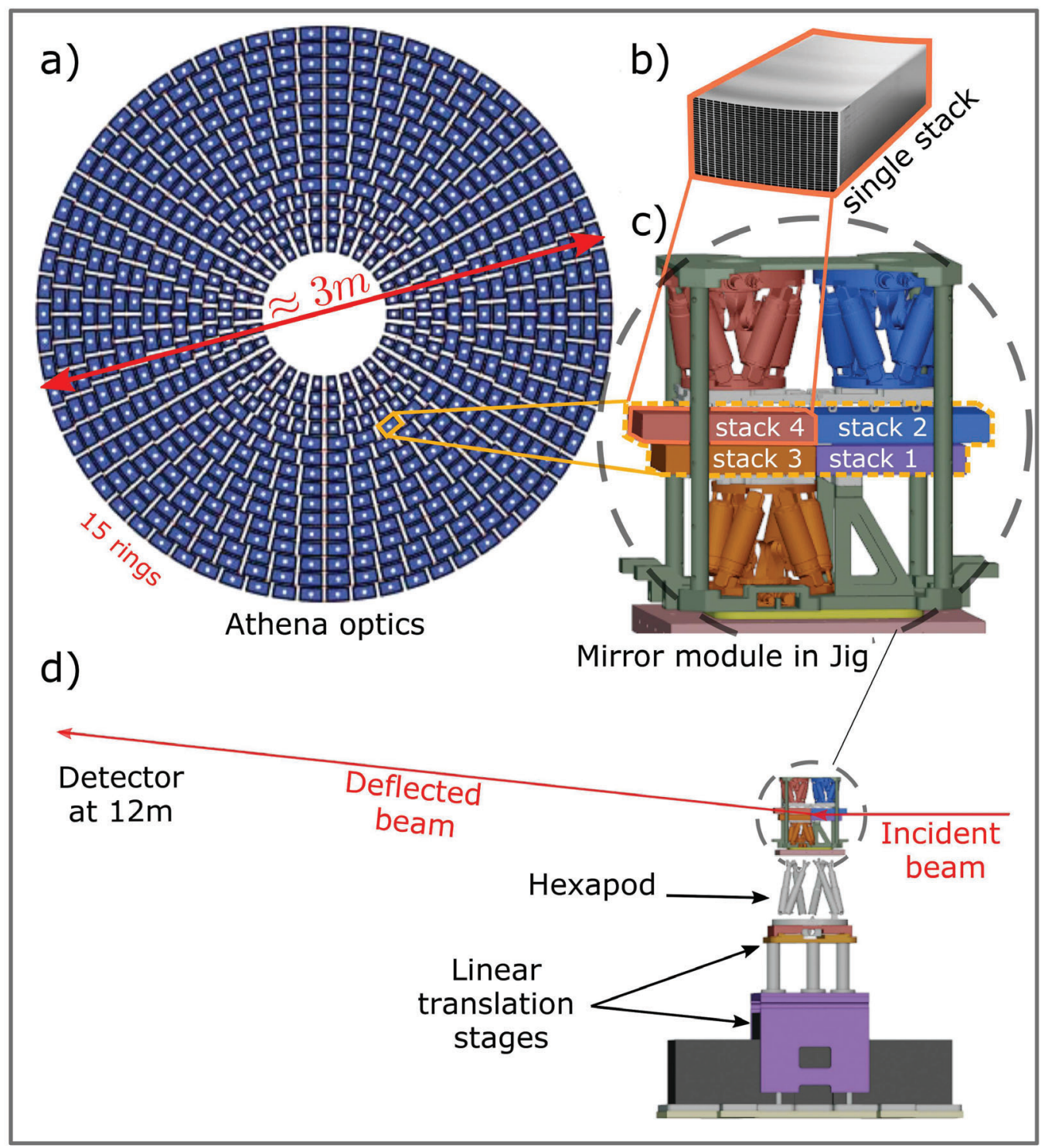

Figure 1. The ATHENA optics made of 15 rings and populated by about 700 mirror modules a). Stack produced by cosine with the Silicon Pore Optics technology b). Mechanical Jig with 4 stacks to be aligned with a collimated X-ray beam c). Principle of measurement of a whole MM at MINERVA d).

precise relative adjustments in position and orientation of an individual stack independently from the others. An $\mathrm{X}$-ray collimated beam is then sent at normal incidence towards the optics, is deflected and partially focused by the MM toward a 2D array detector about $12 \mathrm{~m}$ further (close to the focal plane of ATHENA optics). The Point Spread Function (PSF) is collected and recorded to be analyzed. In order to characterize the whole MM, this last operation is repeated over the entire entrance surface of the optics, and carried out by mechanically moving the MM along a plane perpendicular to the input X-ray beam. At XPBF 2.0, this scan is performed thanks to a larger hexapod where the jig itself is rigidly stuck on its top platform. The data of the PSF pattern generated during the MM scan leads to the computation of the relative geometrical misalignment between the 4 stacks. As a result, a correction is applied on the 3 smaller hexapods and optimization of the position of the 4 stacks within a MM is realized. Characterization of one MM requires usually one day and ATHENA contains about 700 of 
them. For that reason, to boost the assembly process, the 3rd generation synchrotron radiation facility ALBA (Barcelona, Spain) has started the construction of MINERVA, a dedicated test beamline that will provide the necessary equipment to assemble and characterize each MM before mounting on the telescope. MINERVA will be interoperable with XPBF 2.0 and will follow its design as close as possible. ${ }^{7}$ However its construction is also an opportunity to explore new and different approaches to characterize the MMs. This will be reflected later on the different architectures chosen for the MM scanning stage and the detector tower. After briefly presenting the ALBA synchrotron, the main features of MINERVA will be given by describing the concept of its main elements.

\section{THE ALBA SYNCHROTRON}

ALBA is a 3rd generation Synchrotron Light facility located in Cerdanyola del Vallès (Barcelona), being the newest source in the Mediterranean area. It is a complex of electron accelerators to produce synchrotron light, which allows the visualization of the atomic structure of matter as well as the study of its properties. The $3 \mathrm{GeV}$ electron beam energy at ALBA is achieved by combining a LInear ACcelerator (LINAC) and a low-emittance, full-energy Booster placed in the same tunnel as the Storage Ring. ALBA's 268 meter perimeter has 24 straight sections of which 17 are available for the installation of insertion devices. ALBA currently has eight operational state-of-the-art beamlines, comprising soft and hard X-rays, which are devoted mainly to biosciences, condensed matter (magnetic and electronic properties, nanoscience) and materials science. Additionally, four beamlines are in construction (low-energy ultra-high-resolution angular photoemission for complex materials, microfocus for macromolecular crystallography, absorption and diffraction and fast X-ray tomography and radioscopy). The main properties of the ALBA storage ring are listed at table 1. The MINERVA beamline will be fed by a bending magnet source located at port 25.

Table 1. Main parameters of the ALBA Storage Ring.

\begin{tabular}{|l|l|}
\hline Parameter & Value \\
\hline Nominal Energy & $3 \mathrm{GeV}$ \\
\hline Circumference & $268.8 \mathrm{~m}$ \\
\hline Design current & $400 \mathrm{~mA}$ \\
\hline Operating current (Jan 2021) & $250 \mathrm{~mA}$ \\
\hline Energy dispersion & $1.1 \times 10^{-3}$ \\
\hline Emittance & $4.58 \mathrm{~nm} \cdot \mathrm{rad}$ \\
\hline Coupling & $<1 \%$ \\
\hline
\end{tabular}

\section{GENERAL BEAMLINE DESCRIPTION}

As mentioned already earlier, MINERVA will follow the optical layout of XPBF 2.0 and is sketched in Figure 2. It shows the three main components of the beamline: the multilayer monochromator enclosed in the optics hutch of the beamline, the sample chamber inside a temperature-controlled enclosure, and the detector tower. The beamline will take port 25 at the ALBA experimental hall, which provides optimal distribution of the components in the experimental hall, and allows for future upgrades (like possible extension of the MMs focal length). The beamline will operate under Ultra High Vacuum conditions (UHV) from the source to the exit of the photon shutter, where a vacuum window will separate them from the rest of the beamline. Downstream the vacuum window, the beamline will operate under High Vacuum conditions (HV) $\left(10^{-5}\right.$ mbar).

The beamline will provide stable temperature and clean environment around the sample station, and will be equipped with several angle-measuring devices (autocollimators) to control the MMs orientation during measurements. To ensure the accuracy of the geometrical configuration required by the data analysis, the beamline will assess the absolute distance between the fluorescence screen and the mirror module origin. This measurement relies mainly on the performances of laser tracking technology and the high positioning repeatability of the mechanics. For instance, optical targets (cube reflectors) will be firmly fixed to the detector for a continuous read 
out of its position. The main hexapod inside the vacuum chamber will also benefit from laser tracker technology for accurate positioning calibration. In the situation where a single laser tracker is not enough to achieve the required knowledge between the MM and the detector, a second laser tracker might be used close to the sample chamber. The whole beamline will be controlled using the Tango control system ${ }^{8}$, standard at ALBA and many other large instruments facilities. All hardware components will be integrated to the control system, which will provide also continuous monitoring of the Equipment Protection System (EPS) and Personnel Safety System (PSS). The control system will include macro execution and flexible scripting in order to allow for scan automation. ${ }^{9-11}$ Data acquisition will allow recording the detector images together with the necessary configuration parameters of the beamline. In the following sections, details of the main components of the beamline are given.

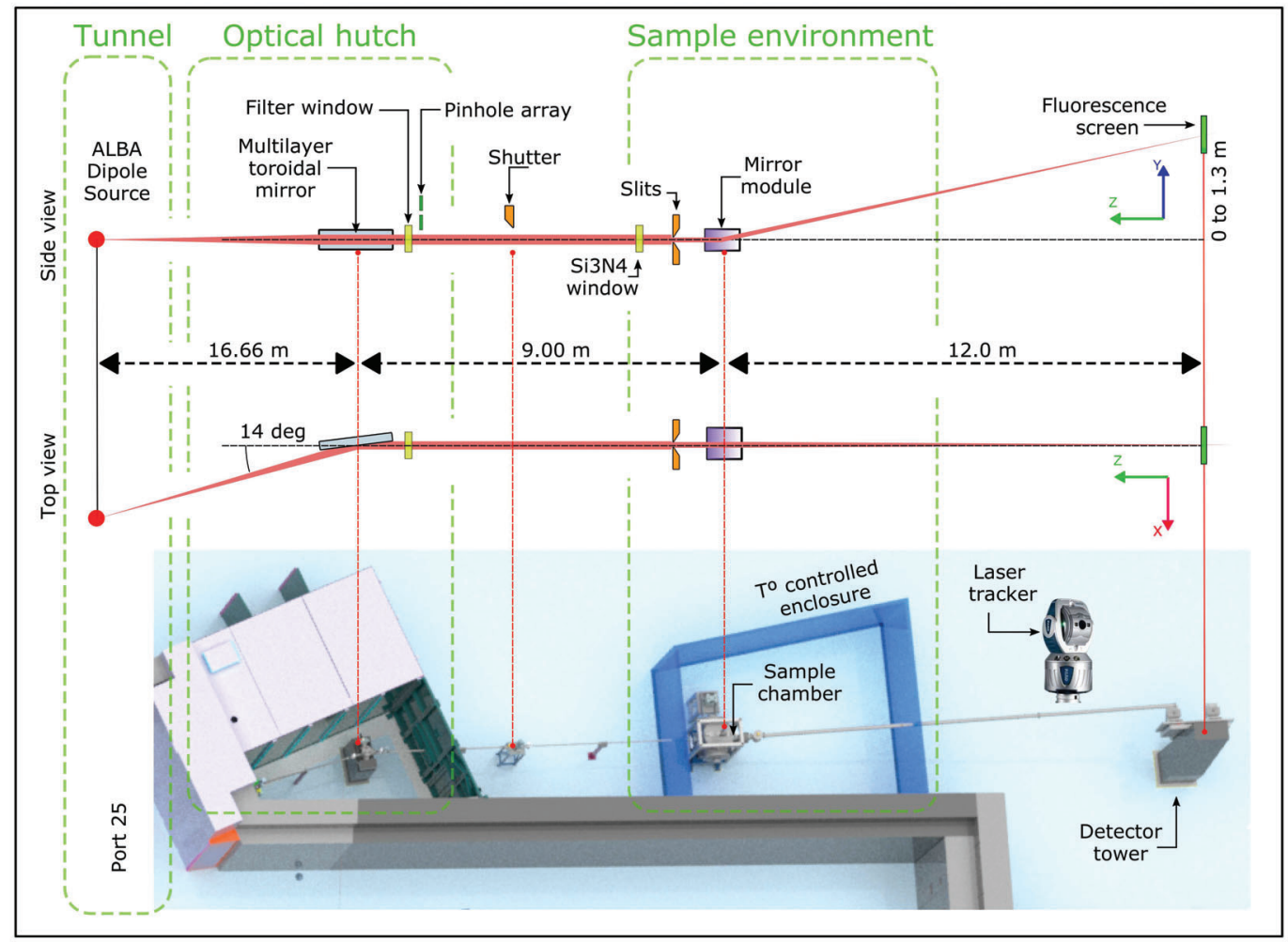

Figure 2. MINERVA layout presenting the main components of the beamline. Side view in a), top view in b) and 3D view in c).

\subsection{Bending magnet and front end}

At MINERVA, the synchrotron radiation is produced by a bending magnet. The characteristics of the source of the electron beam at the extraction point are detailed at table 2 and the spectral distribution of the source for photons in the range 0.5 to $10 \mathrm{keV}$ is given in Figure 3. The angular distribution of emission in the vertical direction is relatively top-flat, because the work energy of the beamline is relatively low with respect to the critical energy of the source. This guarantees a relatively uniform illumination of the sample. The two bumps generated by the vertical polarization of the field emitted at $1 \mathrm{keV}$ contribute to less than $10 \%$ of in-homogeneity.

\subsection{Toroidal mirror, pinhole and optical filters}

The first optical element of the beamline is a multilayer coated toroidal mirror. It deflects the beam horizontally inboard by 14 degrees. The mirror has a toroidal shape in order to collimate the beam emitted by the bending 
Table 2. Source parameters for the beamline.

\begin{tabular}{|l|l|}
\hline Parameter & Value \\
\hline Bending magnet critical energy & $8.499 \mathrm{keV}$ \\
\hline$\gamma$ (Lorentz factor) & 5870.854 \\
\hline Emitted Power (integrated vertically) & $\approx 40 \mathrm{~W}($ at $250 \mathrm{~mA})$ \\
\hline e-beam size $(\mathrm{H} \times \mathrm{V})$ & $46 \times 23.1 \mu \mathrm{m}^{2}(\mathrm{rms})$ \\
\hline e-beam divergence $(\mathrm{H} \times \mathrm{V})$ & $138 \times 2.07 \mu \mathrm{rad}^{2}(\mathrm{rms})$ \\
\hline Photon beam divergence $(\mathrm{H} \times \mathrm{V})$ & $1 \mathrm{mrad} \times 700 \mu \mathrm{rad}(\mathrm{FWHM})$ \\
\hline Spectral Flux $(250 \mathrm{~mA})$ & $1.53 \mathrm{E}+13$ photons $/ \mathrm{s} / 0.1 \mathrm{BW}$ \\
\hline
\end{tabular}

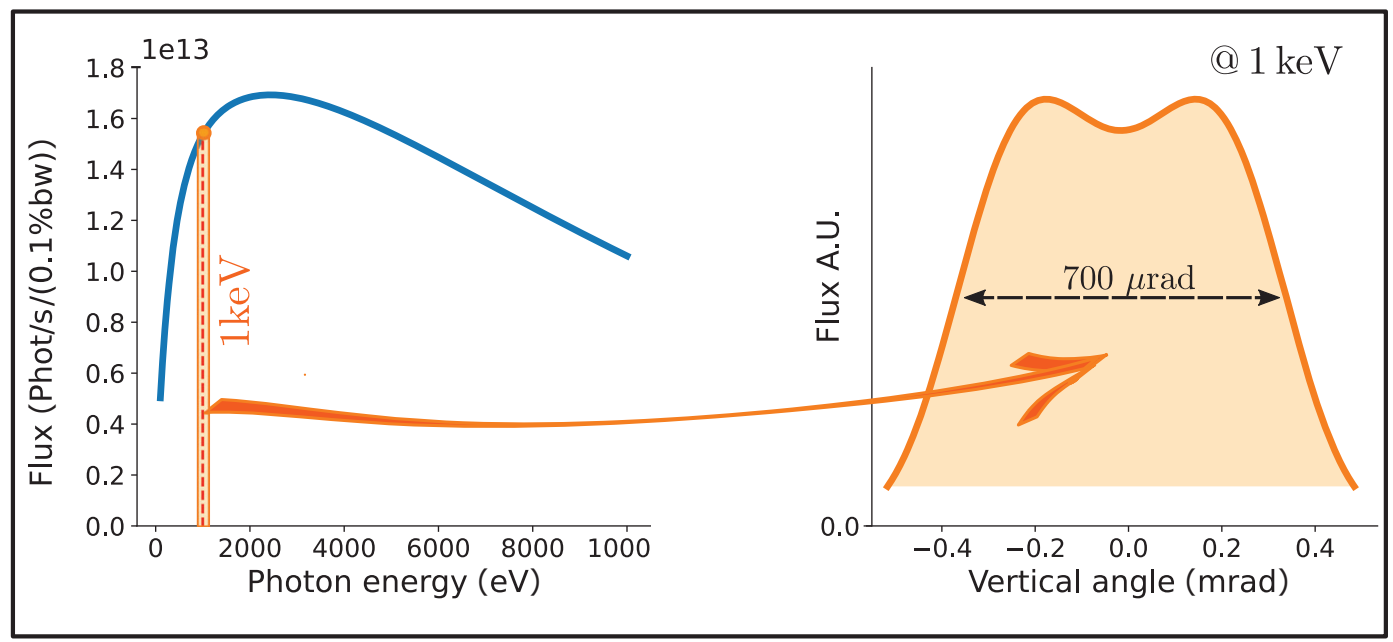

Figure 3. a) Spectral flux delivered by the ALBA bending magnet with 1 mrad horizontal acceptance and $250 \mathrm{~mA}$ electron beam current. The flux at $1 \mathrm{keV}$ is approximately $1.5 \mathrm{e} 13$ photons/sec/0.1\% bw. b) The vertical angular distribution is about $700 \mu \mathrm{rad}$ at $1 \mathrm{keV}$. Calculations performed by XOPPY. ${ }^{12}$

magnet both vertically and horizontally. The calculated spatial distribution after reflection shows a high uniformity around the maximum (Figure 4 a) ) and can be compared with the useful beam dimensions for operation $(8 \mathrm{~mm} \times 8 \mathrm{~mm})$ represented by the dashed green box. The calculated angular distribution is given in Figure 4 b). Calculations were performed assuming a mirror perfectly positioned for collimation. It has been considered a slope error of $1.0 \mu \mathrm{rad} \mathrm{rms}$ in the meridional direction, which contribution remains marginal to the divergence of the beam. In the sagittal direction a slope error eight times larger can be afforded without noticeable effect on collimation. The horizontal and vertical profiles show then a residual divergence of $0.53 \times 0.73 \operatorname{arcsec}^{2} \mathrm{rms}$ for the full illuminated mirror perfectly suitable for the MM characterization.

The mirror surface will be made of a multilayer coating that selects a narrow bandwidth of the incoming radiation about the nominal energy of $1.0 \mathrm{keV}$. As an example, Figure $4 \mathrm{c}$ ) presents the calculated reflectivity for a multilayer constituted by 50 bilayers of Silicon/Tungsten. The parameters (bilayer thickness and $\Gamma$ ) have been chosen to optimize the output at $1 \mathrm{keV}$ (blue curve) and the sharp narrow peak at this photon energy can be observed. The system will reflect also harmonics, but in most of the cases they are filtered by the double reflection in the sample mirror modules themselves. One can also consider an off optimized configuration where the second harmonic is canceled out (red curve). The later solution comes along with an unwanted increase of reflectivity for low energy photons together with an undesirable diminution and broadening of the main reflection peak.

The toroidal mirror will be held in position by a precision positioning system which will provide remote 


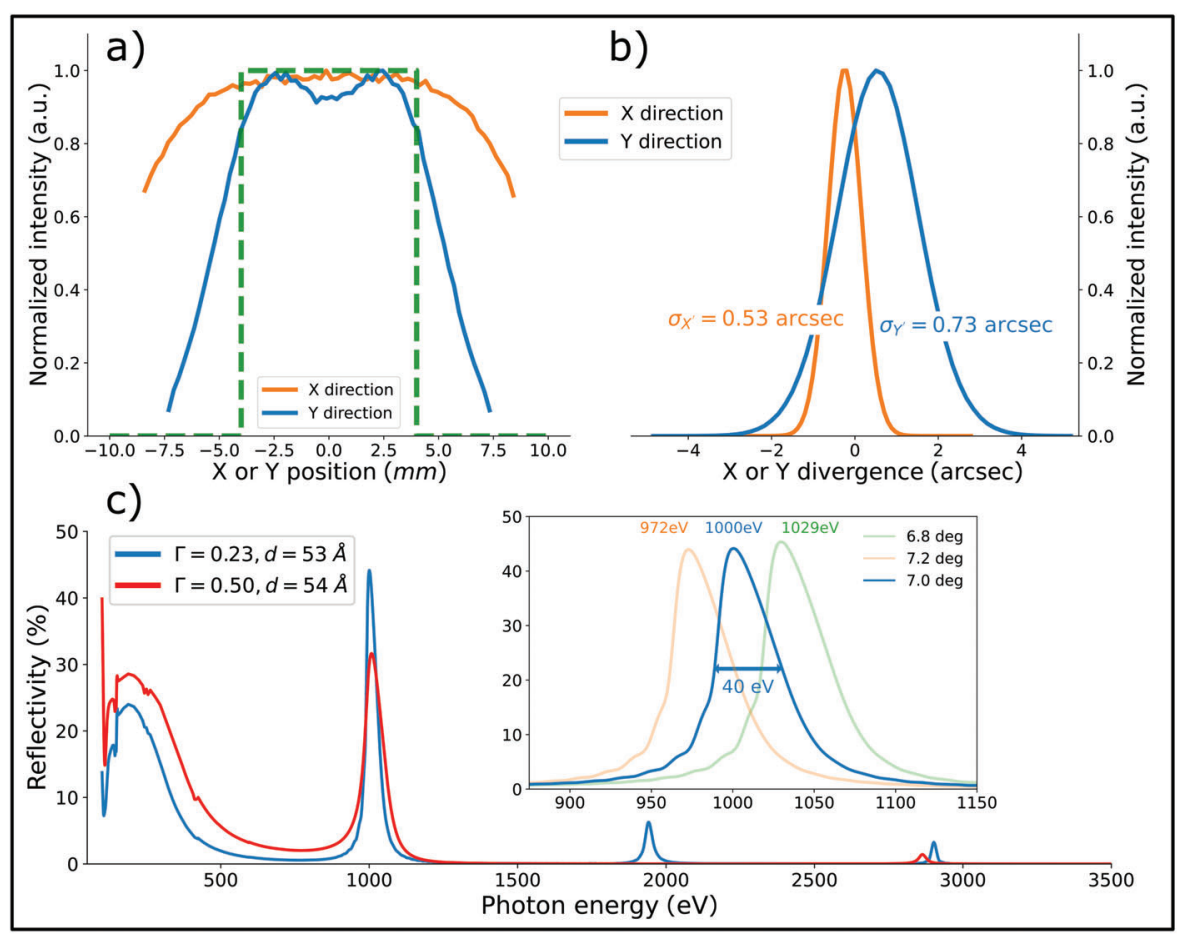

Figure 4. Spatial beam profile in a) and angular distribution in b) of the beam downstream M1 in the horizontal direction (X axis) and vertical (Y axis). Calculations performed with Shadow ${ }^{13}$ are the result of averaging 1000 different random mirror surface with $1.0 \mu \mathrm{rad}$ rms slope error in the meridional direction and $8.0 \mu \mathrm{rad}$ rms slope error in the sagittal direction. Computation of the multilayer reflectivity spectra in c) using 2 different sets of parameters. The blue curve optimizes reflectivity at $1 \mathrm{keV}$ whereas the red curve reduces the 2 harmonic reflection. The later situation is performed by increasing $\Gamma$ and by slightly modifying the bilayer thickness to re-center the main peak at 1 keV. For illustration, the inset plot shows how the main peak shifts only few tens of eV when the mirror is tilted by 200 mdeg, which is comparable to the width of the peak itself (with $\Gamma=0.23$ and $d=54 \AA$ ). Calculations done with XOPPY.

control of the pitch mirror (incidence angle adjustment) and of the x-displacement (along the normal of the mirror surface). The motion will be based on an in-air stepper motor driven mechanics, and will include encoders to provide position feedback on control. The mirror is enclosed in a UHV chamber and will be side cooled by means of a copper water circuit.

In addition to the main opto-mechanical components described above, the MINERVA beamline will include also several apertures, filters and diagnostics. They are briefly described in the following:

- downstream the monochromator, will be placed a pinhole array. It consists on a motorized linear feedthrough that holds a steel plate with pinholes of different diameters. The following diameters are considered: $\varnothing 10$ $\mu \mathrm{m}, \varnothing 20 \mu \mathrm{m}, \varnothing 50 \mu \mathrm{m}, \varnothing 100 \mu \mathrm{m}, \varnothing 200 \mu \mathrm{m}, \varnothing 500 \mu \mathrm{m}$, and fully retracted.

- together with the pinholes, a set of $\mathrm{Si}_{3} \mathrm{~N}_{4}$ membranes is placed to suppress photons with low energy. Indeed, as seen in Figure $4 \mathrm{c}$ ), the multilayer coating reflects a huge amount of low energy photons that has to be cut off. Few hundreds of micrometers of $\mathrm{Si}_{3} \mathrm{~N}_{4}$ will be enough together with the combination of a thin layer of metal ( $\mathrm{Al}$ is considered) to eliminate visible light.

- right upstream the chamber, there is a photon shutter. The shutter block has a pneumatic in/out actuation. The shutter block includes a plate coated with phosphor as screen. When closed, it provides an image of the beam, visible from the viewport that is used for diagnostics. 
- a valve which separates the upstream UHV section from the downstream HV. It consists of a $\mathrm{Si}_{3} \mathrm{~N}_{4}$ membrane with dimensions compatible with a $8 \times 8 \mathrm{~mm}^{2}$ square beam. The window is installed on a gate valve and suppresses low energy photons from the beamline.

- a slits system with four motorized and encoded blades, which can overlap completely and allow for an aperture above $8 \mathrm{~mm}$.

- a manual actuated attenuator which holds several Kapton foils of different thicknesses.

\subsection{Sample environment}

The sample station is located in a clean environment enclosure, which provides also temperature stability. The enclosure will be equipped with an air conditioning system that will stabilize the temperature to a level better than $\pm 1^{\circ} \mathrm{C}$. The setpoint temperature will be set to $20^{\circ} \mathrm{C}$, this is below the nominal temperature of the ALBA experimental hall, for that reason the enclosure will be thermally isolated. Since cleanliness is required for the manipulation of the mirror modules, the air conditioning system of the enclosure will include high-efficiency particulate air filters (HEPA).

The sample vacuum chamber is almost a full replica of the one built at XPBF 2.0 in order to guaranty the maximal compatibility between the two beamlines. The chamber, placed $9 \mathrm{~m}$ downstream the monochromator, is sketched in Figure 5 a). It is large enough to allocate the MM positioning stage (jig) and their alignment mechanics. The vacuum chamber is mechanically decoupled from the sample positioning stages, and it is supported by means of an independent steel frame, fixed directly to the floor. The sample station includes the necessary ports for the stands of the motion system. It accommodates several ports, purposed for electrical or optical fibers feedthroughs, two adapted viewports used by autocollimators and the beam entrance and exit. It incorporates also two large ports with hinged doors at both sides, to allow accessing the sample platform from both sides. It will also include a sliding platform to facilitate the loading and unloading of the jig.

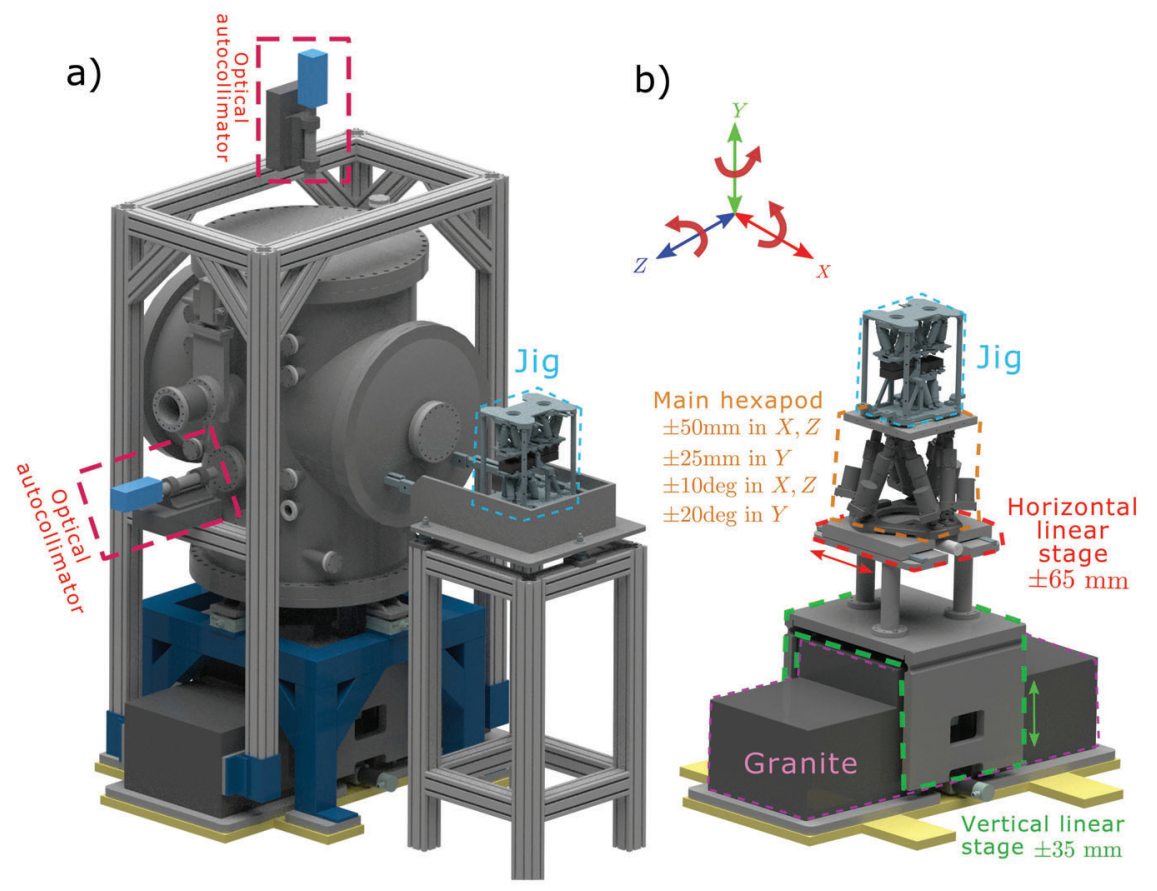

Figure 5. Sample environment. In a) is shown the vacuum chamber surrounded by a aluminum structure supporting the 2 autcollimators. In b ) mechanical solution to limit the number of hexapod orientation corrections.

One of the crucial requirements to characterize a MM is to keep its orientation within 1 arcsec respect to the incident X-ray beam during the whole scanning. The chosen strategy to reach this specification remains identical 
to the one realized at XPBF 2.0. It consists on a control feedback loop based on two optical autocollimators placed around the vacuum chamber (Figure 5 a) and Figure 6 ). The two autocollimators are fixed on the aluminum structure directly sustained by the granite block. They measure the 3 orientation angles of the main hexapod inside the vacuum chamber through viewports with anti-reflective coating layer. Light emitted by the autocollimator get reflected by two large flat mirrors, rigidly attached at the main hexapod platform. If a deviation of more than 1 arcsec with respect to the desired orientation is measured, an algorithm computes the proper adjustments to be applied on the trajectory and acts and the main hexapod moves accordingly (Figure $6)$.

This feedback loop is the most time-consuming step during a complete scan, and the full duration of MM characterization depends substantively on the number of orientation corrections that has been required. For that reason this is one of the few parts that MINERVA differs from the original implementation of XPBF 2.0 aiming at reducing the total number of hexapod re-orientations to be performed by the MM scanning mechanism. The proposed approach consists to delegate the linear scanning motions, initially assigned to the main hexapod, to dedicated linear stages with better straightness. In MINERVA the hexapod will be mounted on top of two high precision linear stages (Figure $5 \mathrm{~b}$ ) ), providing vertical ( $\mathrm{Y}$ axis) and horizontal motion ( $\mathrm{X}$ axis). The whole system is mounted on the top of the granite block. The vertical stage takes place in air and is connected to the rest of the chamber via edge welded bellows. This motion is particularly designed to keep constant the orientation of the MM during a vertical scan thanks to rigid linear guideways fixed on both sides of the granite. The displacement range of this motion is enough to scan the height of a complete MM. On the other hand, both the hexapod and the horizontal linear stage work under vacuum. During standard operations, the horizontal motion purpose is to select the column of pores to be vertically scanned, but the possibility to scan horizontally using this actuator will not be compromised. The displacement range of this motion is enough to scan the width of the largest MM of ATHENA. Since the straightness of the linear stages is better than the one of the hexapod in the full motion range, we expect less requests to the orientation control feedback loop reducing then the overall time to characterize a single MM.

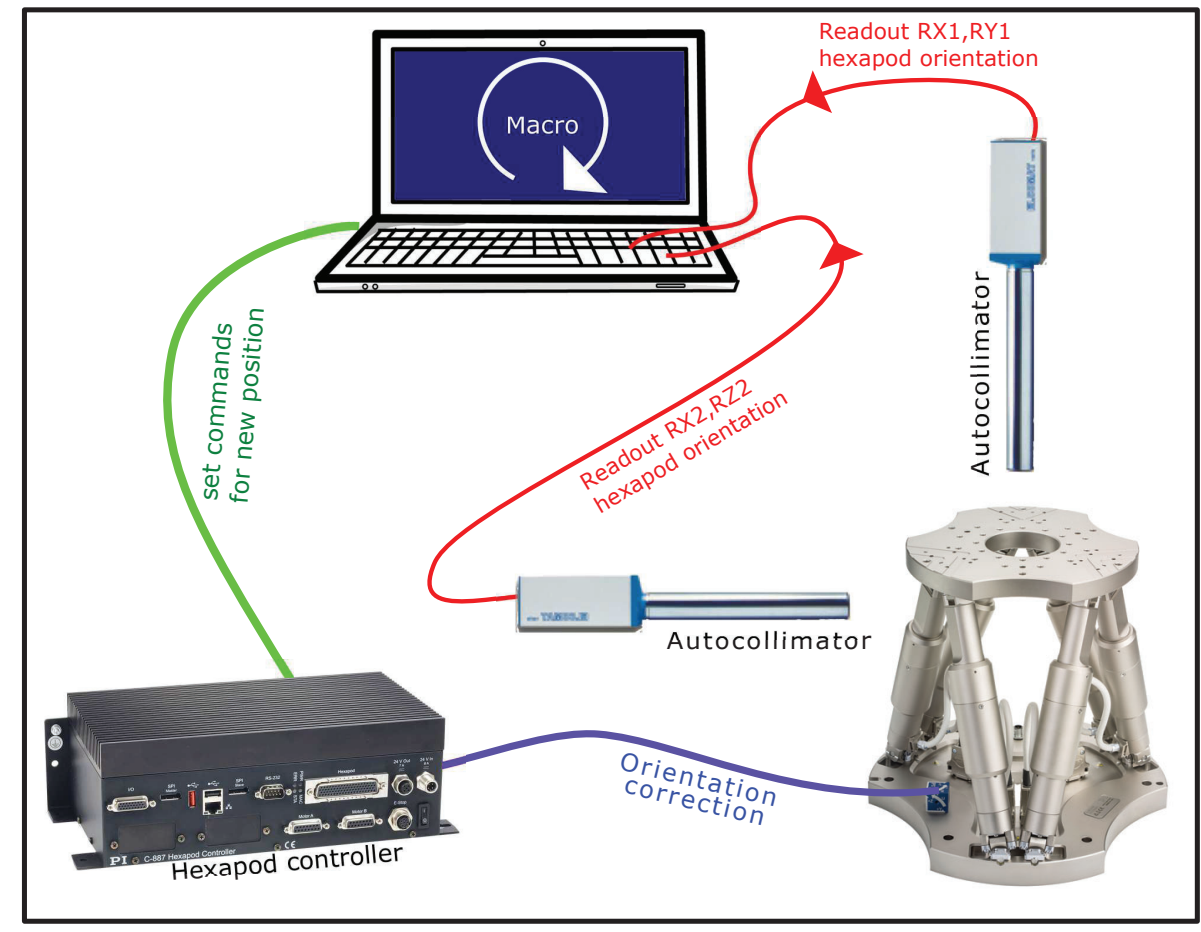

Figure 6. Orientation feedback loop principle realized at XPBF 2.0 and re-used by MINERVA. 


\subsection{Flight tube and Detector tower}

A flight tube links the sample station to the detector and preserves the vacuum along the $12 \mathrm{~m}$ long beam path between the MM and the detector (Figure 7). It can adapt its position to the range of the different deflection angles (its orientation can range from horizontal to about 7 degrees upwards). In addition, its adjustable length can compensate motion of the detector position.

Regarding the imaging detection system, it is entirely based on the one installed at XPBF 2.0. The principle consists first on the conversion of the X-rays into visible light thanks to a phosphor screen. Then follows an optical system (in the visible range and in air) composed by a two lenses optical system to image the screen into the sensor of a 2 dimensional array camera.

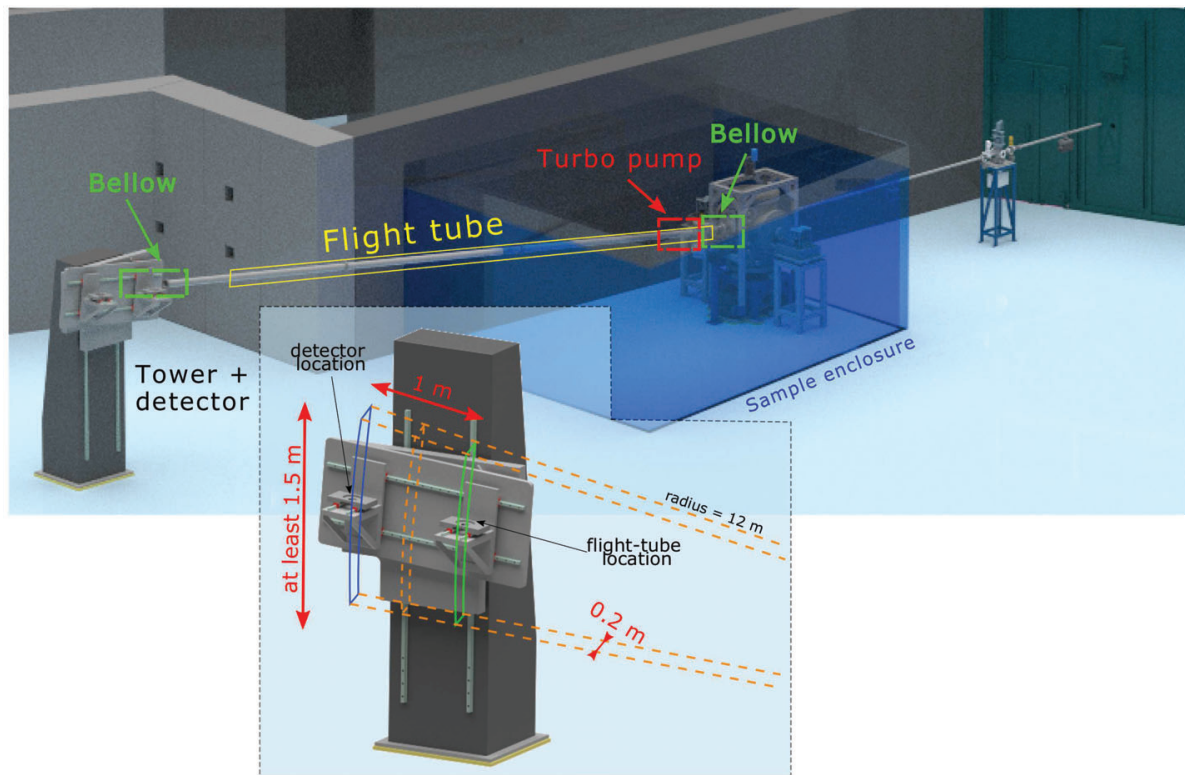

Figure 7. Flight tube connecting the vacuum chamber inside the sample enclosure and the detector tower. The detector positioning mechanism allows all the suitable degrees of freedom to intercept the deflected beam by any kind of MM necessary for the ATHENA optics.

The 2-dimensional array detector will be mounted on a vertical structure (called tower) with enough controlled degrees of freedom to maintain its position in the line of sight of the beam deflected by each MM. In MINERVA, the architecture of the tower deviates also from the one of XPBF 2.0. In Figure 7, is shown the conceptual implementation consisting on a large flat and stable block (steel or granite block) that provides an outstanding reference plane, mechanical and thermal stability. This structure prevents from longitudinal bending developed by the pull forces due to the vacuum inside the flight tube. As seen in the picture, one complete side of the tower is free from any mechanical support allowing a complete visibility to the laser tracker at any positions of the fluorescence screen. All positioning motions will be remotely controllable by stepper motors and relative encoders. In addition to the motion system, the absolute position of the detector will be measured by a laser tracker sitting few meters from the tower. To this end, it will include a frame, rigidly attached to the detector itself, with at least three permanent metrology targets (corner cubes).

\section{CONCLUSION}

The concept of MINERVA has been described, a new beamline under construction at the ALBA synchrotron. The optical layout is a replica of XPBF 2.0 already in operation in the PTB laboratory at BESSY II and used continuously by cosine. However, MINERVA will bring some innovation by trying to reduce the MM characterization time with a different scanning scheme. Also more stability and repeatability are expected with an innovative tower concept bringing enhanced mechanical and thermal stability. All that makes of MINERVA an enhanced instrument for metrology characterization of the ATHENA MMs and further x-rays optical elements. 


\section{REFERENCES}

[1] https://www.cells.es/en.

[2] Krumrey, M., Cibik, L., Müller, P., Bavdaz, M., Wille, E., Ackermann, M., and Collon, M. J., "X-ray pencil beam facility for optics characterization," in [Space Telescopes and Instrumentation 2010: Ultraviolet to Gamma Ray], Arnaud, M., Murray, S. S., and Takahashi, T., eds., 7732, 1517 - 1524, International Society for Optics and Photonics, SPIE (2010).

[3] Nandra, K. et al., "The hot and energetic universe: A white paper presenting the science theme motivating the athena+ mission," (2013).

[4] Collon, M. J., Vacanti, G., Barrière, N. M., Landgraf, B., Günther, R., Vervest, M., Voruz, L., Verhoeckx, S., Babić, L., Keek, L., Girou, D., Okma, B., Hauser, E., Beijersbergen, M. W., Bavdaz, M., Wille, E., Fransen, S., Shortt, B., Ferreira, I., Haneveld, J., Koelewijn, A., Start, R., Wijnperle, M., Lankwarden, J.-J., van Baren, C., Hieltjes, P., den Herder, J. W., Müller, P., Handick, E., Krumrey, M., Bradshaw, M., Burwitz, V., Pareschi, G., Massahi, S., Svendsen, S., Ferreira, D. D. M., Christensen, F. E., Valsecchi, G., Oliver, P., Chequer, I., and Ball, K., "Status of the silicon pore optics technology," in [Optics for EUV, X-Ray, and Gamma-Ray Astronomy IX], O'Dell, S. L. and Pareschi, G., eds., 11119, 174 - 181, International Society for Optics and Photonics, SPIE (2019).

[5] Willingale, R., Pareschi, G., Christensen, F., and den Herder, J.-W., "The Hot and Energetic Universe: The Optical Design of the Athena+ Mirror," (2013).

[6] Vacanti, G., Barrière, N. M., Collon, M. J., Hauser, E., Babić, L., Bayerle, A., Girou, D., Günther, R., Keek, L., Landgraf, B., Okma, B., Verhoeckx, S., Vervest, M., Voruz, L., Bavdaz, M., Wille, E., Krumrey, M., Müller, P., and Handick, E., "X-ray testing of silicon pore optics," in [Optics for EUV, X-Ray, and Gamma-Ray Astronomy IX], O'Dell, S. L. and Pareschi, G., eds., 11119, 149 - 157, International Society for Optics and Photonics, SPIE (2019).

[7] Handick, E., Cibik, L., Krumrey, M., Müller, P., Barrière, N., Collon, M., Hauser, E., Vacanti, G., Verhoeckx, S., Bavdaz, M., and Wille, E., "Upgrade of the x-ray parallel beam facility XPBF 2.0 for characterization of silicon pore optics," in [Space Telescopes and Instrumentation 2020: Ultraviolet to Gamma Ray], den Herder, J.-W. A., Nikzad, S., and Nakazawa, K., eds., 11444, 698 - 705, International Society for Optics and Photonics, SPIE (2020).

[8] https://tango-controls.org.

[9] https://sardana-controls.org.

[10] Coutinho, T., Cuni, G., Fernandez-Carreiras, D., J., J. K., Pascual-Izarra, C., Reszela, Z., Sune, R., Homs, A., Taurel, E., and Rey, V., "Sardana, the software for building SCADAS in scientific environments.," in [Proceedings of ICALEPCS2011], 607 - 609 (2012).

[11] Reszela, Z., Andreu, J., Cuni, G., Coutinho, T. M., Falcon-Torres, C., Fernandez-Carreiras, D., HomsPuron, R., Pascual-Izarra, C., Roldan, D., Rosanes-Siscart, M., de Vera, M. T. N. P., Milan-Otero, A., and Kowalski, G. W., "Generic data acquisition interfaces and processes in Sardana.," in [Proceedings of 17th Int. Conf. on Acc. and Large Exp. Physics Control Systems ICALEPCS2019], 506 - 510, JACoW (2019).

[12] del Río, M. S. and Dejus, R. J., "XOP v2.4: recent developments of the x-ray optics software toolkit," in [Advances in Computational Methods for X-Ray Optics II], del Rio, M. S. and Chubar, O., eds., 8141, 368 - 372, International Society for Optics and Photonics, SPIE (2011).

[13] del Rio, M. S., Canestrari, N., Jiang, F., and Cerrina, F., "SHADOW3: a new version of the synchrotron X-ray optics modelling package," Journal of Synchrotron Radiation 18, 708-716 (Sep 2011). 